\title{
Análisis del proceso de divorcio como transición de salud según la teoría de las transiciones de Alaf Meleis
}

\section{Analysis of the divorce process as health transition according to the theory of Alaf Meleis transitions Análise do processo de divórcio como transição de saúde segundo a teoria das transições de Alaf Meleis}

\author{
Mireia Larrosa Domínguez ${ }^{1}$, Sandra López Estela², Raquel Tejada \\ Musté $^{3}$, Carmen Vives Relats ${ }^{4}$ \\ ${ }^{1}$ Enfermera graduada por la Universitat Rovira $i$ Virgili de Tarragona. Estudiante \\ del Máster en Investigación en Ciencias de la Enfermería en la Universitat Rovira i \\ Virgili. Correo electrónico: mireia.larrosa.dominguez@gmail.com \\ ${ }^{2}$ Enfermera diplomada por la Universitat Rovira $i$ Virgili de Tarragona. Enfermera \\ retén en el Hospital Universitario Joan XXIII de Tarragona. Estudiante del Máster \\ en Investigación en Ciencias de la Enfermería en la Universitat Rovira i Virgili. \\ Correo electrónico: slopezestela@gmail.com \\ ${ }^{3}$ Enfermera diplomada por la Universitat Rovira $i$ Virgili de Tarragona. \\ Especialista en Enfermería Pediátrica por el Ministerio de Educación y Ciencia. \\ Enfermera oncológica en el Hospital de Sant Pau i Santa Tecla de Tarragona. \\ Estudiante del Máster en Investigación en Ciencias de la Enfermería en la \\ Universitat Rovira i Virgili. Correo electrónico: rtmtgn@hotmail.com \\ ${ }^{4}$ Profesora emérita de la Universitat Rovira Virgili. Departamento de enfermería. \\ Correo electrónico: carme.vives@urv.cat
}

\begin{abstract}
Cómo citar este artículo en edición digital: Larrosa Dominguez, M., López Estela, S., Tejada Musté, R. EVives Relats C. (2019). Análisis del proceso de divorcio como transición de salud según la teoría de las transiciones de Alaf Meleis. Cultura de los Cuidados (Edición digital), 23 (54). Recuperado de http://dx.doi.org/10.14198/cuid.2019.54.23

Correspondencia: Carretera Valencia 221 E-1 2. ${ }^{-}-1 .^{\stackrel{a}{ }}$ C.P.: 43006. Tarragona

Correo electrónico de contacto: mireia.larrosa.dominguez@gmail.com Recibido: 30/12/2018; Aceptado: 07/4/2019
\end{abstract}

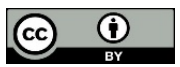

\section{ABSTRACT}

The general goal of this study is to analyze the transitions produced during a divorce process using the Theory of transitions of Alaf Ibraim Meleis. Our specific goals are two:1) Identify the elements that characterize the experience of the divorce transition, and 2) examine if this experience is healthy for the person or not.

A qualitative phenomenological case study was conducted in a person who was experiencing a divorce process. To collect the data, a conversation was conducted in a climate of trust that began with the question: "Are you living through a period of changes?". Free narration and the expression of concerns and feelings were favored. Data processing was done through content analysis of the transcribed conversation, identifying the nature, conditions and 
indicators of the divorce transition.

It is suggested that nursing professionals can and should perform interventions aimed at providing security, self-esteem and confidence to the person experiencing a divorce process. This research concludes that the theory of transitions offers a holistic perspective of the persons experiencing a transition in their lives. It is proposed that the Theory guides the nurse to promote healthy responses to the transition in people.

Keywords: Nursing, transition, divorce, healthy.

\section{RESUMO}

O objetivo geral de nosso estudo é analisar as transições produzidas durante um processo de divórcio, através da Teoria das Transições de Alaf Ibraim Meleis. Como objetivos específicos, propomos a identificação dos elementos que caracterizam a experiência da transição do divórcio e se esta experiência é ou não saudável para a pessoa.

Realizamos um estudo de caso fenomenológico qualitativo em uma pessoa que estava passando por um processo de divórcio. Para coletar os dados, uma conversa foi usada em um clima de confiança que começou com a pergunta: "Você está vivendo um período de mudanças?". A narração livre e a expressão de preocupações e sentimentos foram favorecidas. O processamento dos dados é feito através da análise de conteúdo da conversa transcrita, identificando a natureza, condições e indicadores da transição do divórcio.

Os profissionais de enfermagem podem e devem realizar intervenções que visem proporcionar segurança, autoestima e confiança na pessoa imersa em um processo de divórcio. Conclui-se que a teoria das transições oferece uma perspectiva holística da pessoa que experimenta uma transição em sua vida. Desta forma, a teoria orienta o enfermeiro a promover respostas saudáveis à transição nas pessoas.

Palavras chave: Enfermagem, transição, divórcio saudável.

\section{RESUMEN}

El objetivo general de nuestro estudio es analizar las transiciones producidas durante un proceso de divorcio, mediante la Teoría de las transiciones de Alaf Ibraim Meleis. Como objetivos específicos planteamos la identificación de los elementos que caracterizan la vivencia de la transición del divorcio y si esta vivencia es o no saludable para la persona.

Realizamos un estudio de caso fenomenológico cualitativo en una persona que vivía un proceso de divorcio. Para recolectar los datos se utilizó una conversación en clima de confianza iniciada con la pregunta: "¿Estás viviendo un periodo de cambios?". Se favoreció la libre narración y la expresión de inquietudes y sentimientos. El procesamiento de datos se realiza mediante análisis de contenido de la conversación transcrita, identificando la naturaleza, condiciones e indicadores de la transición de divorcio.

Los profesionales de enfermería podemos y debemos realizar intervenciones dirigidas a proporcionar seguridad, autoestima $y$ confianza en la persona inmersa en un proceso de divorcio. Se concluye que la teoría de las transiciones ofrece una perspectiva holística de la persona que experimenta una transición en su vida. De este modo, la teoría guía a la enfermera a promover en las personas respuestas saludables a la transición.

Palabras clave: Enfermería, transición, divorcio, saludable. 


\section{INTRODUCCIÓN}

Afaf Ibrahim Meleis creó, en relación a la profesión de enfermería, la Teoría de las transiciones en 1971. Definió los componentes, los procesos y las estrategias relacionadas con la suplementación del rol que ayudarían a la persona a realizar una transición saludable.

Las transiciones por las que transcurre la persona y la influencia del entorno pueden ocasionar un desequilibrio entre la demanda y la respuesta ocasionando estrés. De esta manera, cada persona puede responder de diferente manera ante una misma situación a consecuencia de variables ambientales y personales (Lazarus, 1999).

Con intención de aumentar los conocimientos en la investigación en enfermería, Meleis explica que cada cambio en la vida de una persona supone una transición que puede ser de cuatro tipos de transiciones: de desarrollo (incluye aspectos como el nacimiento, crecimiento, madurez, envejecimiento y muerte), situacional, de salud-enfermedad (incluye el proceso de diagnóstico de una enfermedad crónica, alta hospitalaria y recuperación) y organizativo (incluye los cambios en las condiciones del entorno que afectan la vida de los clientes y trabajadores) (IM, 2011).

Meleis determina los conceptos que debemos valorar en la persona que está viviendo un proceso de transición: conciencia, compromiso, cambios y diferencias, evolución en el tiempo, puntos críticos y fenómenos. Dando importancia a la valoración de las condiciones personales, sociales y comunitarias. Además, es importante valorar las transiciones vividas por las personas bajo los supuestos (IM, 2011): son multidimensionales y complejas, se mueven en el tiempo y pueden cambiar roles, relaciones, conductas, habilidades y patrones básicos de la vida.
La insuficiencia de rol vivida por una persona durante un proceso de transición como la situación de divorcio, hace que nos encontremos ante un hecho social que se encuentra ligeramente en aumento y que, por tanto, es importante abordar a muchos niveles. La Real Academia de la Lengua Española define divorcio como "dicho de un juez competente: Disolver o separar, por sentencia, el matrimonio, con cese efectivo de la convivencia conyugal". (Real Academia Española, 2014).

Según datos del Instituto Nacional de Estadística, en el año 2016 se produjeron en España un total de 96.824 divorcios (INE, 25/09/2017). Además, según datos de Idescat, el total de divorcios en Cataluña en el año 2016 fue de 17.931, produciéndose 1.981 de ellos en la provincia de Tarragona (IDESCAT, 2016).

Aunque existen diferentes estudios sobre el impacto que genera el divorcio en la salud física y psicoemocional de la persona adulta (Valdés, Basulto, Choza, 2009; Gómez, 2001), pocos estudios muestran una intervención enfermera que ayude a la persona a sobrellevar de forma efectiva la transición (Castellón, 2000).

El objetivo general de nuestro estudio es analizar las transiciones que se producen durante un proceso de divorcio mediante la Teoría de las transiciones de Alaf Ibraim Meleis. No obstante, los objetivos específicos son identificar los elementos que caracterizan la transición del divorcio en la vivencia de una persona, y conocer si la persona vive la transición del divorcio como un proceso saludable.

\section{MATERIAL Y MÉTODO}

Se realizó un estudio de caso fenomenológico cualitativo en el año 2017. El informante estaba viviendo un proceso de 
divorcio y accedió de manera voluntaria a participar en este estudio. Se produjo un encuentro en su propio domicilio, puesto que es un lugar donde la persona se siente cómoda, tranquila y favorece el feed-back. Se inició la conversación con la pregunta: "¿Estás viviendo un periodo de cambios?". Se favoreció la libre narración de la persona, la expresión de inquietudes y sentimientos, teniendo en cuenta que el simple hecho de narrar la vivencia ya es terapéutico.

Se pretendió detectar la situación de transición por la que estaba pasando la persona, los aspectos que influyen en dicha transición y si ésta es o no saludable. Durante la conversación se negó a ser grabado, por lo que se tomaron notas durante la narrativa para poder analizar los datos posteriormente.

Para garantizar el anonimato de la persona hemos utilizado nombres ficticios, pero las edades y los lugares de residencia son reales.

\section{Presentación del caso}

Javier de 34 años casado desde hace tres años, se encuentra actualmente en un proceso de divorcio debido a diferencias con su pareja e infidelidades por ambas partes. Durante un tiempo han tenido diversas discusiones $y$, aunque intentaron reconciliarse, pronto se dieron cuenta de que no había solución. Además, tienen un hijo de 4 años en común, llamado Marcos.

Su mujer abandonó el domicilio familiar hace seis meses y ya está viviendo con otro hombre. Javier se dedica a trabajar y a cuidar de su hijo, que casi siempre está con él puesto que su ex mujer, por problemas laborales, únicamente puede estar con Marcos en momentos puntuales. De esta manera, cuando Marcos tiene que estar con ella, habitualmente se queda al cuidado de los abuelos maternos.

Javier trabaja a turnos y tiene problemas económicos ya que está endeudado con la hipoteca y el crédito de dos coches. Sin embargo, tiene un nivel adquisitivo medio pero no le es suficiente para hacerse cargo de toda la deuda. Por otra parte, para poder dedicarle más tiempo a Marcos, intenta hacer cambios de turnos con sus compañeros y así trabajar de noche para poder estar con su hijo. Siempre que puede intenta llevar a su hijo a la escuela y disfrutar del tiempo libre con él. Además, Javier cuenta con la ayuda de sus padres y sus dos hermanas que contribuyen al cuidado de Marcos.

La separación está siendo una transición dificultosa, ya que con su ex pareja no llega a un acuerdo sobre el piso y la custodia de Marcos. Además, ella se ha llevado muchísimas cosas del piso, incluidas algunas que no le pertenecían. Javier le ha dado uno de los coches a pesar de estar pagando él mismo el crédito, y ha cedido en varias peticiones que ella le ha hecho para no tener más discusiones ni problemas.

$\mathrm{Al}$ inicio de la separación, Javier pasó por un periodo de tristeza, depresión y pérdida del sentido de vida. Él mismo refiere que "no tenía ganas de comer ni de salir". Además, precisó ayuda psicológica recibiendo tratamiento con ansiolíticos y antidepresivos. Los síntomas se controlaron rápidamente por lo que pudo incorporarse al trabajo en dos meses.

Durante la transición, Javier hizo nuevas amistades y se volcó en su hijo Marcos. Actualmente, se encuentra en pleno divorcio legal con su ex mujer, tiene una nueva pareja con la que mantiene una buena relación y se encuentra feliz. Vive solo en su piso y tiene la custodia compartida de su hijo Marcos.

\section{Análisis del caso. Tipos y patrones de la transición}

La transición que está viviendo actualmente Javier es de carácter 
organizativo ya que se están produciendo cambios en su entorno. La principal transición ha sido la ruptura sentimental que ha desencadenado otra serie de cambios simultáneos en su vida (transiciones), que han afectado a toda la familia.

Su nueva situación ha comportado cambios a nivel social, laboral, emocional. Anteriormente, Javier vivía con su pareja y su hijo Marcos, al que podía ver todos los días. Desde la separación este hecho ha cambiado. Lo ve muy a menudo pero debe compartir la custodia con la madre de su hijo. Y, a pesar de haberles otorgado la custodia compartida, ésta en muchas ocasiones no se cumple puesto que ella no puede hacerse cargo de su hijo por asuntos laborales. De esta manera, Javier puede disfrutar más tiempo de su hijo a pesar de que esto le implica hacer variaciones a nivel laboral. Recientemente ha iniciado una nueva relación sentimental.

En conclusión, Javier está viviendo múltiples transiciones en un mismo periodo, todas ellas secuenciales y a consecuencia de la separación: cambios en la relación padre e hijo, cambios emocionales, cambios del entorno, cambios de rol y cambios a nivel laboral.

\section{Propiedades de la transición}

Conciencia: Javier manifiesta verbalmente que se muestra consciente de los cambios emocionales, laborales, sociales y del entorno sobre la situación percibida como una transición. En un primer momento la respuesta fue negativa ya que, durante un mes manifestó tristeza, depresión, pérdida del sentido de vida y no tenía ganas de salir de casa. Por lo que fue necesario tratamiento con ansiolíticos y antidepresivos. Actualmente, manifiesta encontrarse más animado desde que ha creado un nuevo vínculo de amigos y tiene una nueva pareja.
Compromiso: Javier, al ser consciente del proceso de transición, presenta un alto grado de implicación. Se muestra muy atento con su hijo e intenta estar todo el tiempo posible con él. Además, intenta aprender a cocinar y a realizar las tareas domésticas.

Cambios y diferencias: Tras la separación se ha producido un cambio en la relación con su ex pareja. Anteriormente, vivían juntos y tenían una buena relación. Actualmente la relación no es buena.

Tiempo: El proceso de separación y divorcio de Javier, tuvo su inicio hace seis meses tras la percepción de un cambio en su vida. Desde el inicio hasta la actualidad, ha tenido diferentes emociones que han llegado a producirle sintomatología ansiosodepresiva. En un primer momento, tuvo un período de inestabilidad y confusión (se sintió perdido, triste, no encontraba salida a la situación y pensaba que su problema no tenía solución). Actualmente, aún se encuentra en proceso de divorcio pero se siente más recuperado y está iniciando una relación sentimental y está luchando por conseguir la estabilidad.

Puntos críticos y fenómenos: Están relacionados con el abandono del domicilio por parte de su ex mujer, la relación tormentosa con ella y la custodia de su hijo Marcos. En la actualidad, Javier tiene visión de futuro ya que ha iniciado una nueva relación sentimental.

\section{Condiciones de la transición}

Condiciones personales: Javier ha tenido varias rupturas sentimentales a lo largo de su vida, pero con ninguna de ellas tuvo hijos y únicamente con una de ellas compartió vivienda. Sin embargo, refiere que la separación fue percibida de manera negativa ya que tuvieron varios desacuerdos. El hecho de tener un 
antecedente previo de separación difícil y, además teniendo ahora un hijo en común con su exmujer, hace que su vivencia actual sea mucho más negativa.

Condiciones sociales o comunitarias: Sus padres y sus hermanas cuidan de su hijo cuando él trabaja. Además, su madre le prepara la comida cada día y en ocasiones le compra alimentos. Por ese motivo, podemos decir que Javier tiene un adecuado soporte social y afectivo por parte de diferentes miembros de su familia. Por otro lado, su actual pareja le está aportando estabilidad y tiene una muy buena relación con él y su hijo Marcos, hecho al que él da mucha importancia.

Observamos cómo estos cambios en la vida de Javier se iniciaron como un proceso de transición no saludable, con sentimientos negativos, precisando ayuda profesional y farmacológica. Sin embargo, gracias a los procesos y estrategias relacionados con la suplementación del rol, Javier ha conseguido afrontar el cambio llegando a alcanzar una transición saludable.

Tiene unas condiciones personales $\mathrm{y}$ familiares que favorecen este cambio. Los principios y creencias vinculados a su cultura le han ayudado a hacer frente esta situación y desarrollar mecanismos adecuados para la transición. De este modo, aunque tiene problemas económicos ha preferido seguir teniendo su propio piso y no volver a vivir con sus padres. Sin embargo, acepta la ayuda de su familia durante esta transición. Por otro lado, aunque precisa cambios a nivel laboral para poder conciliar la vida familiar, tanto sus compañeros como la directiva de la empresa le proporcionan muchas facilidades para poder llevarlos a cabo.

\section{Patrones de respuesta o indicadores de proceso de salud en la transición}

Podemos identificar que Javier es plenamente consciente de los cambios que está viviendo durante este periodo, tanto a nivel social, laboral, familiar, etcétera. Él mismo ha sido capaz de definir el momento en que el proceso de transición no era saludable, y solicitar ayuda cuando ha sido conveniente. De esta manera, la ayuda tanto de su entorno como de los profesionales sanitarios ha influido positivamente en la evolución del proceso, para conseguir una transición saludable.

Javier muestra una actitud positiva frente el cambio. Intenta mejorar la situación mediante un análisis de su transición para encontrar soluciones efectivas.

\section{Intervención terapéutica de enfermería}

En los procesos de transición observados en Javier, los profesionales de enfermería debemos realizar intervenciones con el objetivo de fortalecer la seguridad, autoestima y confianza. En este sentido, la conversación con la enfermera es productiva, ya que favorece la expresión de sentimientos positivos y negativos respecto la circunstancia que está viviendo Javier en la actualidad.

El hecho de que la persona narre permite expresar aquello que siente, que vive y que piensa, siendo la narrativa en sí terapéutica. Es importante animar a Javier a continuar con la terapia psicológica para favorecer la expresión de sentimientos, emociones $\mathrm{y}$ búsqueda de estrategias. Además, debemos favorecer una correcta adherencia al tratamiento farmacológico recomendado. Por último, debemos animar a Javier a que realice actividades recreativas y de ocio con su nueva pareja y amigos.

Dentro de nuestras intervenciones enfermeras, podríamos reunirnos con los 
padres de Javier, con el fin de conocer su opinión sobre la situación y trabajar sus emociones. No obstante, se proporcionará recomendaciones con el fin de ser un buen soporte para Javier y Marcos, ya que, para esta transición, la familia es importante para él.

Por último, es importante conocer la vivencia de Marcos en relación al proceso de divorcio de sus padres. Para ello, podríamos animarlo a que hiciera un dibujo y determinar así, sus sentimientos y emociones.

\section{CONCLUSIONES}

Cuando los profesionales de enfermería detectamos la vivencia de una transición, en este caso el divorcio, debemos proporcionar a la persona educación sanitaria con el fin de promover la salud y prevención de la enfermedad, para conseguir una transición saludable. Con el mismo objetivo, debemos ofrecer también las herramientas y estrategias de afrontamiento necesarias en estas situaciones.

Creemos que es importante abordar el fenómeno del divorcio además de legalmente, a nivel sanitario, ya que influye negativamente en la vida de las personas pudiendo causar repercusiones a nivel de salud, produciendo estrés, pánico, problemas de organización, dificultades para la socialización, etcétera.

La teoría de las transiciones nos ofrece una perspectiva holística acerca de la persona que está experimentando una transición en su vida. De esta manera, la teoría guía a la enfermera a promover respuestas saludables a la transición.

\section{BIBLIOGRAFÍA}

- Castellón, A. M. D. (2000). El rol de enfermería frente a una estructura familiar cambiante. Investigación y educación en enfermería, 18(1), 87-92. Recuperado de http://www.redalyc.org/articulo.oa?id=105218294006.

- Gómez-Díaz, J. A. (2011). Fenomenología del divorcio (o la esencia de la separación) en mujeres. Psicologia \& Sociedade, 23(2), 391-397. Recuperado de http://www.scielo.br/pdf/psoc/v23n2/a20v23n2.pdf.

- Raile Alligood, M. \& Marriner Tomey, A. (2011).Teorías y modelos en enfermería, Barcelona: Elsevier.

- Instituto de Estadística de Cataluña (2016). Idescat. Anuario estadístico de Cataluña. Nulidades, separaciones y divorcios. Provincias. Recuperado de https://www.idescat.cat/.

- Instituto Nacional de Estadística. (2017). INEbase/Sociedad/Seguridad y justicia/Estadística de nulidades, separaciones y divorcios/Resultados. Recuperado de http://www.ine.es/.

- Lazarus, R.S. (1999). Estrés y emoción. Manejo e implicaciones en nuestra salud. Bilbao: Desclée De Brouwer.

- Real Academia Española. (2014). Diccionario de la Real Academia Española de la Lengua. Recuperado de http://dle.rae.es/.

- Valdés, Á. A., Basulto, G., y Choza, E. (2009). Percepciones de mujeres divorciadas acerca del divorcio. Enseñanza e investigación en psicología, 14(1). Recuperado de http://www.redalyc.org/articulo.oa?id=29214102. 